\title{
Auto-Avaliação e Avaliação por Pares - Estratégias para o Desenvolvimento Profissional do Médico
}

\author{
Self and Peer Assessment - Strategies for \\ the Professional Development of the \\ Physician
}

Rosângela Curvo Leite Domingues ${ }^{1}$

Eliana Amaral ${ }^{2}$

Angélica Maria Bicudo Zeferino ${ }^{3}$

PALAVRAS-CHAVE:

- Auto-Avaliação;

- Aprendizagem;

- Comissão de Avaliação de Exercício Profissional.

KEY-WORDS:

- Self-assessment;

- Learnig;

- Peer Reviews.

Recebido em: 18/07/2006

Aprovado em: 14/03/2007

$173 \mid \begin{array}{ll}\text { REVISTA BRASILEIRA DE EDUCAÇÃO MÉDICA } \\ 31(2)-17-17 ; 207\end{array}$
${ }^{1}$ Departamento de Tocoginecologia, Faculdade de Ciências Médicas, Universidade de Campinas, Campinas,Brasil.

${ }^{2}$ Departamento de Tocoginecologia, Faculdade de Ciências Médicas, Universidade de Campinas, Campinas, Brasil.

${ }^{3}$ Departamento de Pediatria, Faculdade de Ciências Médicas, Universidade de Campinas,Campinas, Brasil. 
Os currículos mais atualizados na área de saúde visam preparar não apenas médicos qualificados, mas também alunos autônomos, capazes de atuar em ambientes complexos e dinâmicos e de lidar com a crescente quantidade de informações e situações profissionais a que estão expostos ${ }^{1,2}$. A escola médica deve engajar os alunos em um processo contínuo de aprendizagem, no qual habilidades reflexivas, analíticas e de automonitoramento são fundamentais ${ }^{3}$.

Ao longo dos anos, a avaliação em educação médica tem sido uma responsabilidade essencialmente do corpo docente. No entanto, na perspectiva de capacitar médicos para desenvolvimento contínuo e atuação em equipes, há uma crescente tendência de responsabilização dos estudantes, incluindo o papel de agentes de sua própria avaliação e da avaliação de seus pares ${ }^{4}$. Os educadores têm buscado dados colhidos de múltiplas fontes, criando um "círculo completo" de avaliadores, que inclui professores, colegas, enfermeiros, outros profissionais e pacientes, além do próprio aluno ${ }^{5-7}$.

A auto-avaliação representa uma estratégia de ensino importante que prepara o aluno para repensar os resultados de suas próprias ações profissionais; refletir sobre o que aprendeu; avaliar como tal aprendizado o preparou para realizar as tarefas esperadas; perceber suas necessidades individuais de aprendizagem; elaborar um plano coerente para lidar com suas dificuldades; comparar os novos resultados com os anteriores e revisar e atualizar seu plano de aprendizado $^{3,8}$. Essa habilidade de identificar valores e atitudes pessoais, reconhecendo os próprios pontos fortes e fracos, pode ser desenvolvida, aperfeiçoada e modificada pela educação, sendo considerada essencial para a manutenção e melhoria da proficiência médica, para o bom relacionamento com pacientes e colegas, e para o desenvolvimento da identidade profissional $3,5,9,10$.

No entanto, apesar de sua reconhecida importância no processo de aprendizagem contínua dos profissionais da área de saúde, a auto-avaliação tem sido negligenciada ${ }^{11}$. Em parte, isso ocorre porque nem os docentes e nem os alunos parecem estar adequadamente preparados para a transição entre a abordagem de avaliação centrada no professor e a centrada no aluno. Parece que o momento ideal para essa transição é quando os alunos iniciam o estágio clínico do curso médico, pois eles começam a adquirir experiência em cuidar dos pacientes e podem usar esses encontros como guia de avaliação de seus próprios conhecimentos e habilidades. Uma vez conscientes de suas dificuldades, eles poderão decidir como irão usar o tempo limitado que possuem para atividades de autoaprendizagem ${ }^{12}$. Atividades de preparação quando os alunos vão iniciar o estágio clínico, facilitando o desenvolvimento de seu autoconhecimento e encorajando-os a aprender a partir de seus erros, são estratégias pedagógicas que devem ser incorporadas aos currículos médicos ${ }^{3}$.

Diversos autores defendem o uso da auto-avaliação em conjunto com a avaliação feita por pares e professores $4,6,13$ 16. A comparação entre esses dois métodos de avaliação fornece aos educadores informações úteis para o desenvolvimento e planejamento do currículo e para a revisão da metodologia adotada, além de estimular a discussão e o feedback 5,17 .

A avaliação pelos colegas talvez seja o método informal mais usado pelos médicos ${ }^{13}$. A Abim (American Board of Internal Medicine) incorporou a avaliação feita por pares no processo de recertificação de profissionais e no treinamento de futuros profissionais ${ }^{5,18}$. Como os docentes não estão presentes $100 \%$ do tempo, eles podem perder informações importantes a respeito do desempenho do aluno. Por isso, ouvir a perspectiva de colegas com quem o aluno convive por um longo período de tempo e em diferentes situações pode levar a mudanças positivas de comportamento, especialmente no que se refere à competência humanista6,19-22. Entretanto, o conceito dado pelos colegas pode sofrer o chamado "efeito halo", isto é, pode ser influenciado mais pelas impressões gerais que o avaliador tem do avaliado do que pelo desempenho do mesmo. Sabe-se, porém, que esse efeito pode ser reduzido quando se tem uma amostra adequada de avaliadores, dados confidenciais e um instrumento estruturado ${ }^{21,23}$.

\section{CONCLUSÃO}

A capacitação do aluno para ser agente atuante em seu desenvolvimento profissional é uma questão central da educação médica. Neste contexto, tanto a auto-avaliação quanto a avaliação feita por pares parecem ser métodos promissores. No entanto, é preciso compreender a relutância dos alunos em avaliarem seus pares e a si mesmos e desenvolver programas que preparem alunos e professores para realizarem avaliações de forma consciente e construtiva, criando paralelamente um ambiente propício e seguro de avaliação. Deve-se acompanhar a auto-avaliação do aluno ao longo do curso, comparando-a com as avaliações que ele recebe dos colegas e docentes. Cabe, então, às escolas médicas se mobilizarem e criarem condições adequadas para que estudantes e docentes consigam criar uma nova cultura de avaliação, como instrumento de aprendizado e crescimento profissional. Para tanto, a auto-avaliação e a avaliação pelos pares precisam ser integradas no conjunto de instrumentos necessários ao desenvolvimento do profissional médico. 


\section{REFERÊNCIAS}

1. Mattheos N, Nattestad A, Falk-Nilsson E, Attstrom R. The interactive examination: assessing students' self-assessment ability. Med Educ. 2004; 38(4):378-89.

2. Armstrong E, Parsa-Parsi R. How can physicians' learning styles drive educational planning? Acad Med. 2005; 80(7):680-4.

3. Henderson $\mathrm{P}$, Johnson $\mathrm{MH}$. An innovative approach to developing the reflective skills of medical students. BMC Med Educ. [online]. 2002 [capturado 12 maio 2006]; Disponível em: http://www.biomedcentral.com/1472-6920/2/4.

4. Van der Vleuten CPM, Schuwirth LWT. Assessing professional competence: from methods to programmes. Med Educ. 2005; 39(3): 309-17.

5. Rudy DW, Fejfar MC, Griffith CH, Wilson JF. Self- and peer assessment in a first-year communication and interviewing course. Eval Health Prof. 2001; 24(4): 436-45.

6. Davis JD. Comparison of faculty, peer, self and nurse assessment of obstetrics and gynecology residents. Obstet Gynecol. 2002; 99(4): 657-51.

7. Rodgers KG, Manifold C. 360-degree feedback: possibilities for assessment of the ACGME core competencies for emergency medicine residents. Acad Emerg Med. 2002; 9(11):1300-4.

8. Murray E, Gruppen L, Catton P, Hays R, Woolliscroft JO. The accountability of clinical education: its definition and assessment. Med Educ 2000; 34(10):871-9.

9. Woolliscroft JO, Tenhaken J, Smith J, Calhoun JG. Medical students' clinical self-assessments: comparisons with external measures of performance and the students' selfassessments of overall performance and effort. Acad Med. 1993; 68(4): 285-94.

10. Fitzgerald JT, White CB, Gruppen LD. A longitudinal study of self-assessment accuracy. Med Educ. 2003; 37(7): 645-9.

11. Belar C, Brown RA, Hersch LE, Hornyak LM, Rozensky $\mathrm{RH}$, Sheridan EP, et al. Self-assessment in clinical health psychology: a model for ethical expansion of practice. Prof Psychol Res Pr. 2001; 32(2):135-41.

12. Gruppen LD, White C, Fitzgerald JT, Grum CM, Woolliscroft JO. Medical students's self-assessments and their allocations of learning time. Acad Med. 2000; 75(4): 374-9.

13. Ramsey PG, Wenrich MD, Carline JD, Inui TS, Lasrson EB, LoGerfo JP. Use of peer ratings to evaluate physician performance. JAMA. 1993; 269(13):1655-60.

14. Epstein RM, Hundert, EM. Defining and assessing professional competence. JAMA. 2002; 287(2): 226-35.
15. Norman GR, Shannon SI, Marrin ML. The need for needs assessment in continuing medical education. BMJ. 2004; 328(7446): 999-1101.

16. Wilkinson TJ, Frampton CM. Comprehensive undergraduate medical assessments improve prediction of clinical performance. Med Educ. 2004; 38(10):1111-6.

17. Southgate L, Hays RB, Norcini J, Mulholland H, Ayres B, Woolliscroft J, et al. Setting performance standards for medical practice: a theoretical framework. Med Educ. 2001; 35(5): 474-81.

18. Thomas PA, Geb KA, Hellmann DB. A pilot study of peer review in residency training. J Gen Inter Med. 1999;14(9): 551-4.

19. Van Rosendaal GMA, Jennett PA. Comparing peer and faculty evaluations in an internal medicine residency. Acad Med. 1994; 69(4): 299-303.

20. Sullivan ME, Hitchcock MA, Dunnington GL. Peer and selfassessment during problem-based tutorials. Am J Surg. 1999; 177(3): 266-9.

21. Norcini JJ. Peer assessment of competence. Med Educ. 2003; 37(6): 539-43.

22. Evans R, Elwyn G, Edwards A. Review of instruments for peer assessment of physicians. BMJ. 2004; 328(7450): 1240-3.

23. Arnold L. Assessing professional behavior: yesterday, today and tomorrow. Acad Med. 2002; 77(6): 502-15.

\section{Conflito de Interesse.}

Declarou não haver.

\section{Endereço para correspondência}

Rosângela Curvo Leite Domingues

Rua Luciano Venere Decourt, 858 - Cidade Universitária 13083-740 - Campinas - SP

e-mail: rosangela@cpdec.com.br 